\title{
Recurrent networks expect
}

\author{
Adam Ponzi , Jeffery R Wickens \\ From 24th Annual Computational Neuroscience Meeting: CNS*2015 \\ Prague, Czech Republic. 18-23 July 2015
}

How the brain anticipates future events is one of the most intriguing questions in neural information processing. The brain does not simply respond to the external world but in predictably structured environments like natural language, birdsong and somatosensory flow in fluids predicts it [1]. Reactions are improved by predictive encoding of what sensory stimuli may occur as well as when they may occur. Quantitative studies of implicit timing in streaming perceptual discrimination tasks show that performance is enhanced if stimuli occur at expected times according to an established rhythm. The neural basis for implicit timing is not fully understood but oscillatory entrainment mechanisms have been suggested [2]. Recent studies confirm that low-frequency brain oscillations do become phase entrained in such tasks. Phase entrainment increases with the temporal regularity of the sensory stream and correlates with enhanced discrimination performance [3]. It is not limited to sensory cortices; larger scale cortical networks, as well as subcortical networks like the basal ganglia [4], may also be coherently modulated by the predictability of stimuli streams. The presence of large scale oscillatory entrainment suggests that network mediated neuronal ensemble dynamics may be involved. Recurrent neural networks generate complex but reproducible temporally extended dynamical activity patterns in response to input stimuli [5,6]. Such transient activity patterns have been suggested to provide a natural substrate for working memory and a representation of elapsed time.

Here we add to the understanding of how random recurrent neural networks support neural information processing by demonstrating that temporal expectation also naturally emerges from their dynamics. We show that the weakly chaotic oscillations generated by recurrent networks can be phase synchronized [7] by

\footnotetext{
* Correspondence: adamp@oist.jp

Okinawa Institute of Science and Technology Graduate University (OIST), Onna, Okinawa 904 0495, Japan
} 
3. Cravo AM, Rohenkohl G, Wyart V, Nobre AC: Temporal expectation enhances contrast sensitivity by phase entrainment of low-frequency oscillations in visual cortex. J. Neurosci 2013, 33(9):4002-4010.

4. Geiser E, Notter M, Gabrieli JD: A corticostriatal neural system enhances auditory perception through temporal context processing. J. Neurosci 2012, 32(18):; 6177.

5. Buonomano DV, Merzenich MM: Temporal information transformed into a spatial code by a neural network with realistic properties. Science 1995, 267:1028-1030.

6. Ponzi A, Wickens JR: Optimal Balance of the Striatal Medium Spiny Neuron Network. PLoS comp bio 2013, 9(4):e1002954.

7. Pikovsky A, Zaks M, Rosenblum M, Osipov G, Kurths J: Phase synchronization of chaotic oscillations in terms of periodic orbits. Chaos 1997, 7(4):680-687.

doi:10.1186/1471-2202-16-S1-P285

Cite this article as: Ponzi and Wickens: Recurrent networks expect. BMC Neuroscience 2015 16(Suppl 1):P285.

\section{Submit your next manuscript to BioMed Central} and take full advantage of:

- Convenient online submission

- Thorough peer review

- No space constraints or color figure charges

- Immediate publication on acceptance

- Inclusion in PubMed, CAS, Scopus and Google Scholar

- Research which is freely available for redistribution

Submit your manuscript at www.biomedcentral.com/submit 